\title{
Recurrence Quantification Analysis of Lorenz Model Periodically Modulated at the Onset of Chaos
}

\author{
P. BABINEC* AND A. KRAFČÍK \\ Department of Nuclear Physics and Biophysics, Faculty of Mathematics, Physics, and Informatics \\ Comenius University, Mlynská dolina F1, 84248 Bratislava, Slovakia
}

(Received May 18, 2010; in final form April 29, 2011)

\begin{abstract}
Plots and quantitative measures obtained from recurrence quantification analysis - recurrence rate, determinism, entropy, and trapping time, were used for the sensitive detection of transitions from laminar (regular) to turbulent (chaotic) phases in the Lorenz model periodically modulated at the onset of chaos.
\end{abstract}

PACS: 05.40.-a, 87.10.Mn, 43.60. $+\mathrm{d}, 43.71 .+\mathrm{m}$

\section{Introduction}

Recurrence, i.e. ability of dynamical system to return to the vicinity of the initial point in phase space is considered to be one of the most basic attributes of a deterministic process since its introduction by Poincaré [1]. More than 20 years ago the method of recurrence plots (RPs) was proposed by Eckmann, Kamphorst, and Ruelle [2]. RPs are constructed on the basis of mutual distances between points belonging to the same trajectory. More precisely, RP for a time series of $N$ points $x_{i}$ ( $i$ is time index) is matrix $N \times N$ filled with white and dark points, where a dark point, called recurrence point is put at the position of coordinates $(i, j)$ provided that their distances $\left\|x_{i}-x_{j}\right\|$ is smaller than some threshold value $\varepsilon$. Despite the simplicity, the success of RPs relies on the fact that their structures are visually appealing and already have been widely applied in diverse areas [3-6].

\section{Principle of recurrence plot method}

In Figs. 1 and 2 RPs calculated using CRP Toolbox for Matlab [7] corresponding to deterministic as well as random signals are shown. We have used signal of the type $x=(1-a) s+a \xi$, where $s$ is the periodic sawtooth signal and $\xi$ is the Gaussian white noise. Purely random time series exhibit stuctureless RPs, whereas recurrent patterns, especially lines, or zebra skin like formations parallel to the main diagonal of the plots, correspond to deterministic dynamics in the time series. In order to obtain quantitative measures recurrence quantification analysis (RQA) was developed [8]. In this study only four of them are used. The first measure of RQA is recurrence rate $(\mathrm{RR})$ which measures density of recurrence points in $\mathrm{RP}$

\footnotetext{
* corresponding author; e-mail: babinec@fmph.uniba.sk
}

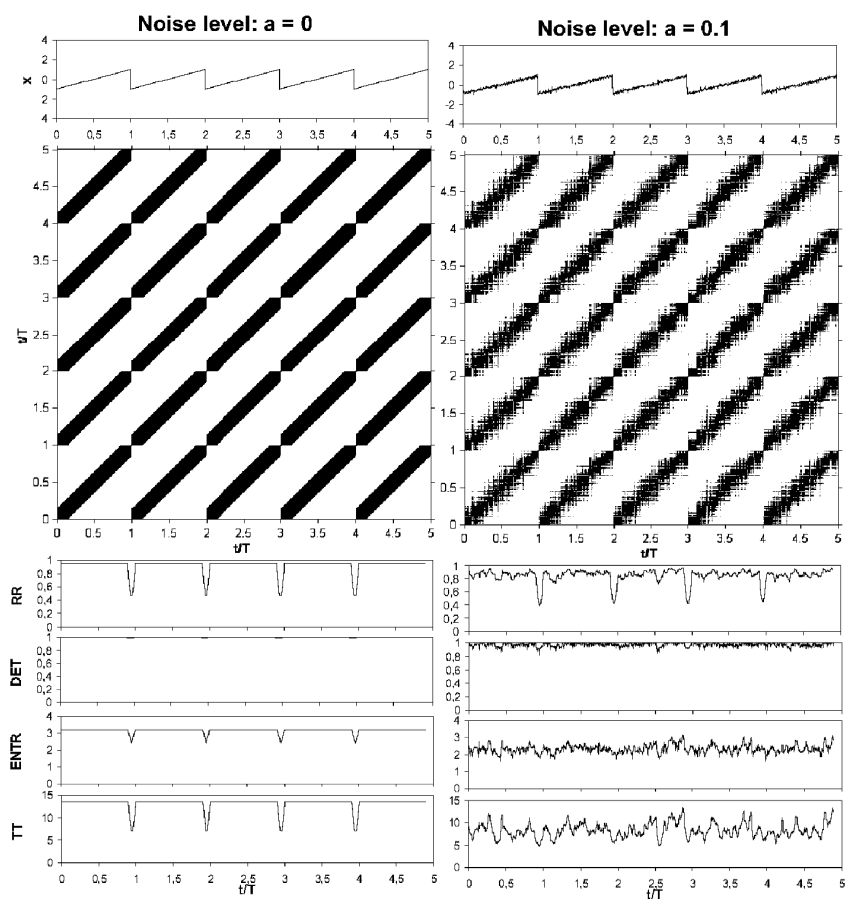

Fig. 1. Recurrence plot corresponding to signals with increasing degree of noise: noise level $a=0$ (purely deterministic sawtooth signal) and signal with noise level: $a=0.1$; with results of windowed recurrence quantification analysis (embedding dimension $=1$; delay $=1$; neighborhood $=0.5 ;$ time window size $=(1 / 10)(t / T)$ and window shift $=1$ ).

$$
\mathrm{RR}=\frac{1}{N^{2}} \sum_{i, j=1}^{N} R_{i, j}(\varepsilon),
$$

where $R_{i, j}(\varepsilon)=\Theta\left(\varepsilon-\left\|x_{i}-x_{j}\right\|\right)$, with $\Theta(\cdot)$ the Heaviside function and $\|\cdot\|$ - a selected norm.

Chaotic processes are characterized with none or short diagonals, deterministic processes, on the other hand, cause longer diagonals. The ratio of recurrence points 


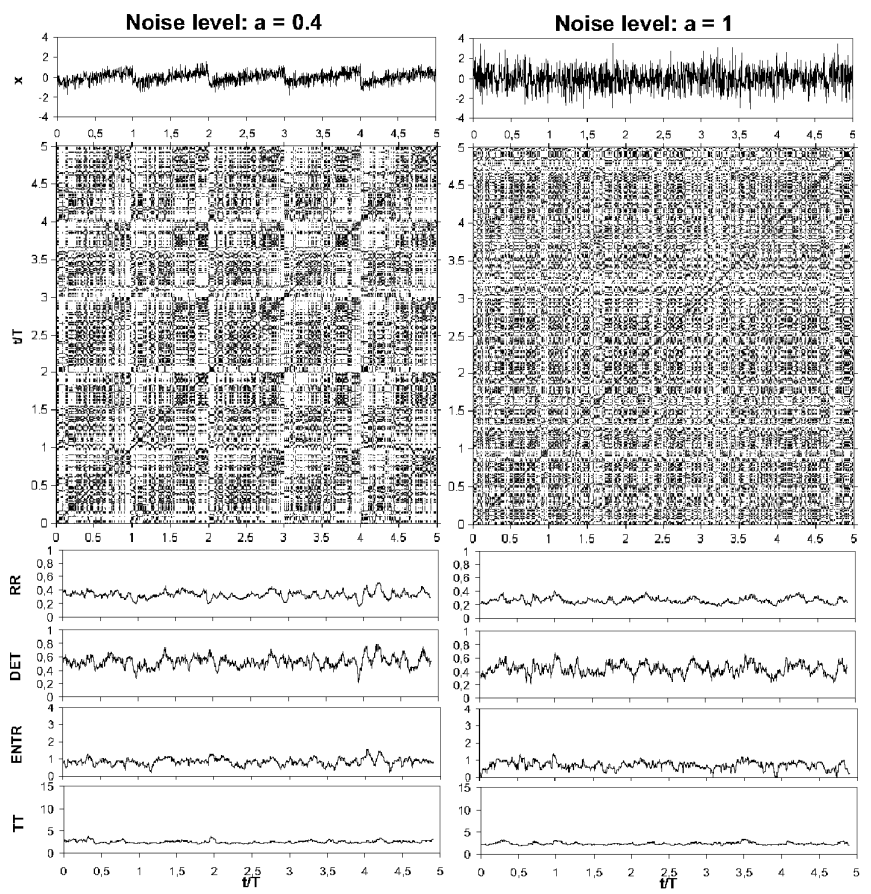

Fig. 2. Recurrence plot corresponding to signals with increasing degree of noise: noise level $a=0.4$ and pure white noise (noise level: $a=1$ ); with results of windowed recurrence quantification analysis (embedding dimension $=1$; delay $=1$; neighborhood $=0.5$; time window size $=(1 / 10)(t / T)$ and window shift $=1)$.

that form diagonal structures (of at least length $l_{\text {min }}$ ) to all recurrence points represents measure known as determinism

$$
\mathrm{DET}=\frac{\sum_{l=l_{\min }}^{N} l P(l)}{\sum_{l=1}^{N} l P(l)},
$$

where $P(l)$ is number of diagonal lines with lengths $l$.

Third measure - entropy corresponds to the Shannon entropy of probability $p(l)=P(l) / N_{l}$ to find a diagonal line of length $l$ in $\mathrm{RP}$

$$
\mathrm{ENTR}=-\sum_{l=l_{\min }}^{N} p(l) \ln p(l) .
$$

The last measure which would be used in this paper is trapping time which corresponds to the average length of vertical lines

$$
\mathrm{TT}=\frac{\sum_{v=v_{\min }}^{N} v P(v)}{\sum_{v=1}^{N} P(v)} .
$$

\section{Results and discussion}

The Lorenz model, which is subject of this study [9] is described by the equations

$$
\frac{\mathrm{d} x}{\mathrm{~d} t}=10(y-x),
$$

$$
\begin{aligned}
& \frac{\mathrm{d} y}{\mathrm{~d} t}=-x z+R x-y, \\
& \frac{\mathrm{d} z}{\mathrm{~d} t}=x y-\frac{8}{3} z,
\end{aligned}
$$

where coordinate $x$ measures the rate of convective (vertical) turnover, $y$ - the horizontal temperature variation, and $z$ - the vertical temperature variation. The parameter $R$ is proportional to the Rayleigh number, and we will follow the study of Crisanti et al. [10] where this parameter has a periodic time variation

$$
R=R_{0}-A \cos \left(\frac{2 \pi t}{T}\right) .
$$

Periodic variations mimic the seasonal changing of the solar heat input. We have used $R_{0}=25.5$ close to the threshold $R_{\mathrm{Cr}}=24.74$ and $A=4$, for the transition from stable fixed points to a chaotic attractor. When the control parameter changes periodically in time, below and above the threshold for the onset of chaos, stochastic resonance effect appears, producing alternations of chaotic and regular phases [10]. Because the Lyapunov exponent for this system is positive, the existence of regular predictable phases is rather controversial. As has been shown by Aurell et al. [11], single global Lyapunov exponent is not able to characterize the above behavior, since it refers to a very long time property of the system, and therefore introduced local average of the exponential rate of divergence for initially close trajectories, as a more suitable parameter.

Our aim in this study is to analyze periodically modulated Lorenz model using windowed RQA. From initial time series $\left(x_{1}, x_{2}, \ldots, x_{n}\right)$, when $x_{i}=i \tau_{\mathrm{s}}, \tau_{\mathrm{s}}=$ sampling time, we form an $N$-point long series

$$
\begin{aligned}
& E_{1}=\left(x_{1}, x_{2}, \ldots, x_{N}\right) \\
& E_{2}=\left(x_{1+w}, x_{2+w}, \ldots, x_{N+w}\right) \\
& \vdots \\
& E_{\mathrm{P}}=\left(x_{1+(P-1) w}, x_{2+(P-1) w}, \ldots, x_{N+(P-1) w}\right),
\end{aligned}
$$

where $w$ is the offset, and the number of epochs (windows) satisfied the relation $N+(P-1) w \leq n$.

Figure 3 shows the results obtained for shorter time period $T=100$ (left column) when the probability of switching between regular and chaotic intervals is very small and longer period $T=1000$, where this switching occurs with probability close to 1 (stochastic resonance). The dynamical behavior of system is easy to understand from the visual inspection of RPs, and this is in accord with quantitative RQA parameters. It can be seen that the most significant changes are observed for trapping time TT, which was introduced into RQA only recently [12].

\section{Conclusion}

From the example of periodically modulated Lorenz model it is clearly evident that RQA parameters are 


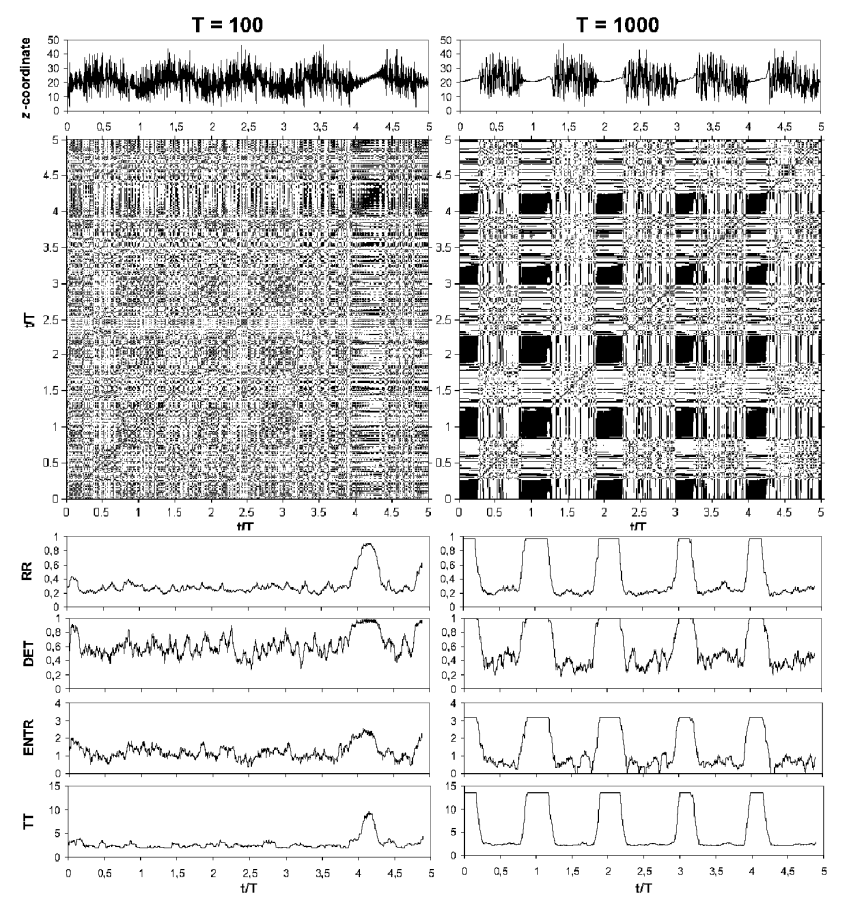

Fig. 3. Recurrence plots and results of windowed recurrence quantification analysis (embedding dimension $=1 ;$ delay $=1 ;$ neighborhood $=0.5$; time window size $=(1 / 10)(t / T)$ and window shift $=1)$ corresponding to $z$-coordinate of the Lorenz model modulated with a periodic signal with period $T=100$ (left) and period $T=1000$ (right).

able to detect efficiently and more straightforwardly than rather complicated local Lyapunov exponent [10] the crossover between the deterministic and chaotic dynam- ics by analysis of only one measured observable, e.g. the instantaneous temperature in meteorology or currency exchange rate in finance.

\section{Acknowledgments}

This work was supported by VEGA grant 1/0642/11.

\section{References}

[1] H. Poincaré, Acta Math. 13, 1 (1890).

[2] J.-P. Eckmann, S.O. Kamphorst, D. Ruelle, Europhys. Lett. 4, 973 (1987).

[3] N. Marwan, M.C. Romano, M. Thiel, J. Kurths, Phys. Rep. 438, 237 (2007).

[4] P. Babinec, L. Zemanová, M. Babincová, Phys. Med. 18, 63 (2002).

[5] P. Babinec, J. Leszczynski, Chaos Solitons Fractals 17, 981 (2003).

[6] L. Matassini, H. Kantz, J. Holyst, R. Hegger, Phys. Rev. E 65, 021102 (2002).

[7] N. Marwan, CRP Toolbox 5.2, (2007). http://tocsy.agnld.uni-potsdam.de/crp.php .

[8] C.L. Weber Jr., J.P. Zbilut, J. Appl. Physiol. 76, 965 (1994).

[9] E.N. Lorenz, J. Atmos. Sci. 20, 130 (1963).

[10] A. Crisanti, M. Falcioni, G. Paladin, A. Vulpiani, J. Phys. A 27, L597 (1994).

[11] E. Aurell, G. Boffetta, A. Crisanti, G. Paladin, A.N. Vulpiani, J. Phys. A 30, 1 (1997).

[12] N. Marwan, N. Wessel, U. Meyerfeldt, A. Schirdewan, J. Kurths, Phys. Rev. E 66, 026702 (2002). 